\title{
Powder Characterization by Particle Shape Assessment ${ }^{\dagger}$
}

\author{
Paramanand Singh \\ Dept. of Metallurgical Engineering I.I.T., * \\ P. Ramakrishnan \\ Dept. of Metallurgical Engineering I.I.T., **
}

\begin{abstract}
In addition to the process variables, the quality of the powder metallurgical products depends to a large extent on the materials, bulk properties and morphological characteristics of the powders. Material characteristics are relatively well established but the same is not the case with the other two variables because unequivocal definitions of some of the morphological characteristics like particle shape, size and texture on which the bulk properties are strongly depended are yet to be evolved. Among the morphological characteristics, particle shape, though one of the most investigated area remains the least understood. In spite of the complexity of the problem, the necessity for obtaining a better insight of concerned branches in which powders are extensively used have yielded many dimensional and dimensionless parameters for characterizing the powders based on particles shape. The present paper classifies the available literature on the particle shape into four main categories, briefly describes their salient features, critically assesses the merits and demerits of each class and thus makes the perspective more clear for proper and judicious application of one or more methods, of course, depending on the level of accuracy required for charactering the powders.
\end{abstract}

\section{Introduction}

In powder metallurgy (P/M), characterization of the raw materials which are in the powdered state is a sine qua non prerequisite to ensure quality, reproductivity and low scatter of the properties of the sintered parts, but interestingly enough, one of the most important variables over which the P/M parts' manufacture has very little control is: the characteristics of the powder itself. Fundamentally, the characteristics of the powder are influenced by the material, method of powder production and the process variables in a particular method. These basic variables of powder production collectively give rise to plethora of characteristics, the knowledge of which becomes essential for powder characterization in course of its journey to the end product. Hausner ${ }^{1}$, in an attempt to demonstrate the intricacy of the problem of powder characterization, records as many as seventeen characteristics of a powder particles and twelve characteristics of mass of powder and mentions that several of the characteristics are hard to define and even more difficult to determine. Thus, visualizing the dire necessity of powder characteriza-

\footnotetext{
* Madras 600036 , India

** Bombay 400 076. India

† Received 27 April, 1996
}

tion and at the same time the manifold problems involved therein, it becomes incumbent upon the researchers to dive deep and take up this challenging task. Considerable research has been going on in this direction. To maneuver the complex situation of powder characterization more pragmatically, characteristics of powder which influence its behaviour during handling and processing have been divided under two broad categories as shown in Figure $\mathbf{1}$.

Of these, material characteristics are well-understood and almost completely known since chemistry and physics of the material are highly developed areas, but the same is not the case with the morphological characteristics. It is an underdeveloped and underexplored area, and unequivocal and unambiguous definitions of some of the morphological parameters are yet to be evolved ${ }^{2}$. These ill-defined and even less-understood morphological features interact in a complex way, each one of them contributing varying degree of influence to the total behaviour of the powder mass.

Thus, in the light of the above prologue, the present work critically assesses the relevant knowledge pertaining mostly to the morphological features of the powder particles for the progress of better understanding in handling the powder with more confidence. 


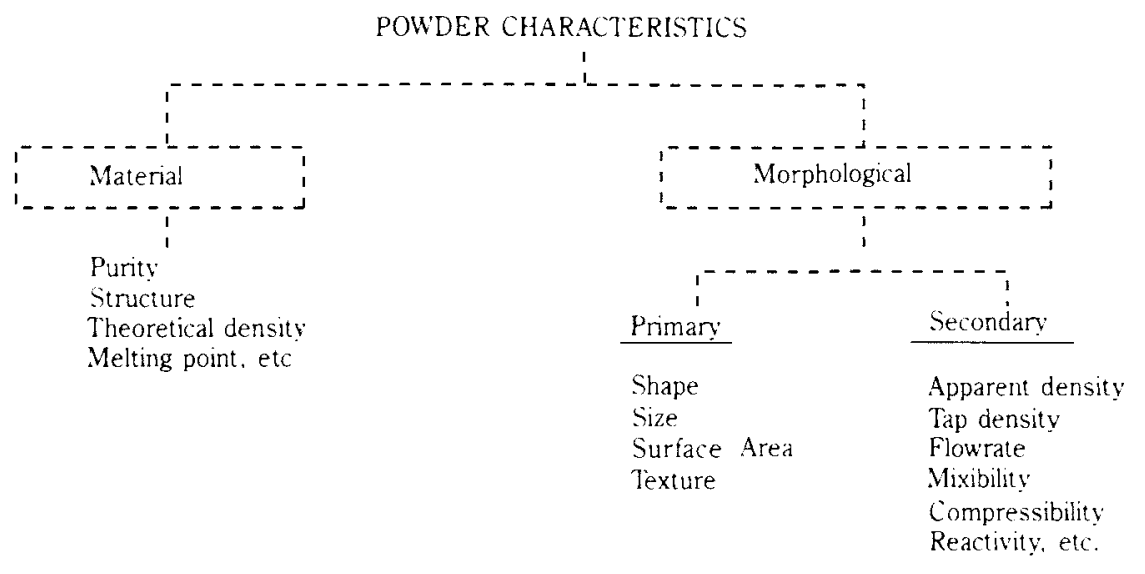

Fig. 1 A broad classification of powder characteristics.

\section{Definition of shape and type of powder particle}

Powder particle is a very complex entity. Its complexity depends upon the material as well as the process of production. It can be a smooth (even) and completely solid tiny mass or rough (jagged) and spongy in nature and hence, numerous categories are possible depending upon its external and internal details ${ }^{3}$.

Shape is the expression of only external morphology of the powder particle and it includes form (overall shape), roundness (smoothness) and surface texture $^{4}$. Figure 2 and $\mathbf{3}$ depict this definition of shape. Mathematically speaking, shape is the recognised pattern of relationships among all of the points which constitute the external surface ${ }^{2}$, i.e., complete description of shape requires the specification of a closed curve in space. Implementation of such a rigorous definition of shape for fine powder particles is difficult and complex and hence, in most of the situations a three dimensional particle is projected in a plane to obtain two dimensional closed curve and then this closed curve is taken to describe the particle shape. Based on the nature of the particle profile in two dimensional plane, particle can be put under two classes - Analytic or holomorphic particle and nonanalytic or nonhomorphic particle 2 . Analytic or holomorphic particle is defined such that a vector from the centre of gravity of the particle to any point on the particle surface intersects the surface but once. This is pictorially represented in Figure 4(a) and 4(b). For example, gas atomized copper powder and to a large extent reduced copper powders belong to this category. Nonanalytic or nonholomorphic particle is defined such that at least one radius vector from the centre of gravity of the particle

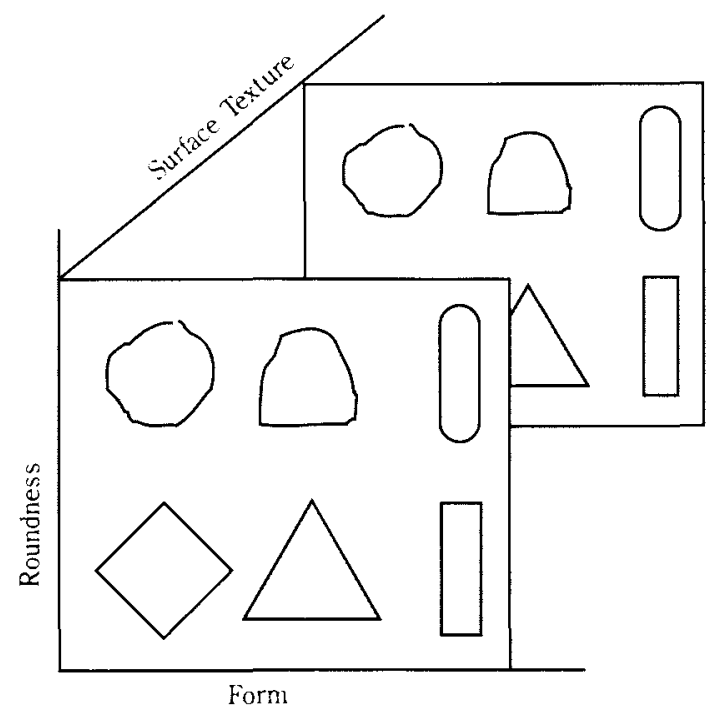

Fig. 2 Representation of form, roundness and surface texture by three linear dimensions to illustrate their independence.

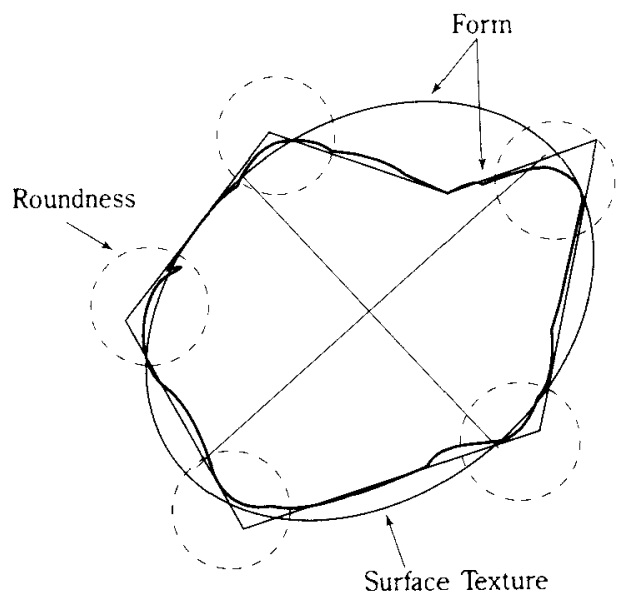

Fig. 3 A particle outline (heavy solid line) with its component elements dimensions to illustrate their independence. 


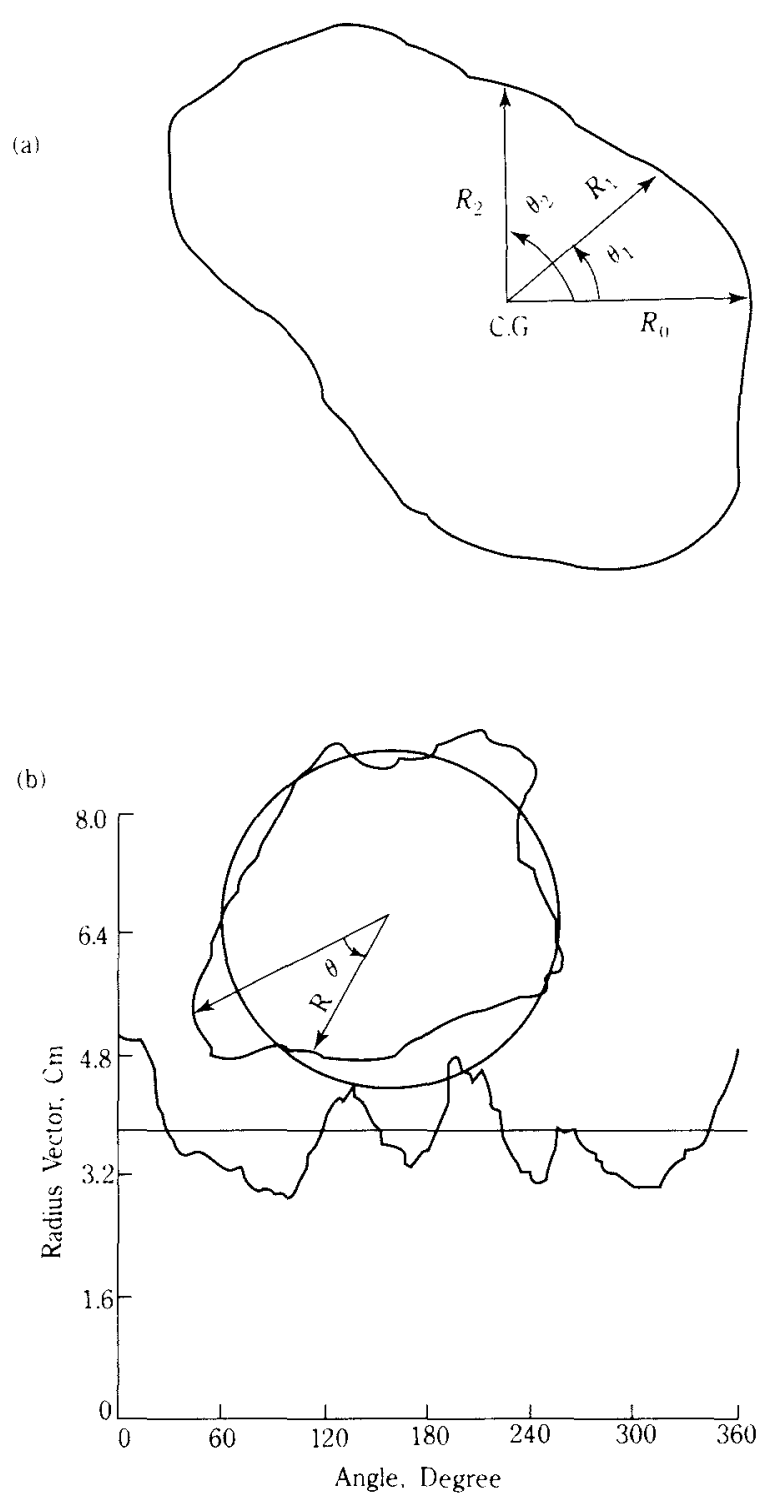

Fig. 4 (a) and (b) are two different ways of representing holomorphic or analytic particle profile.

intersects the surface of particle more than once. This is pictorially represented in Figure 5(a) and 5(b). Electrolytic copper powder and carbon black aggregates belong to this category.

The objective of the preceding definition of shape and classification of the particle types is to provide the basis for critically examining the merits and demerits and also of the level of accuracy of a particular approach for powder characterization reviewed under the subsequent subsections.

\section{Panoramic view of powder characterization}

Size and shape are fundamental characteristics of a powder particle and hence, of a given mass of powder. In fact, both size and shape are intimately

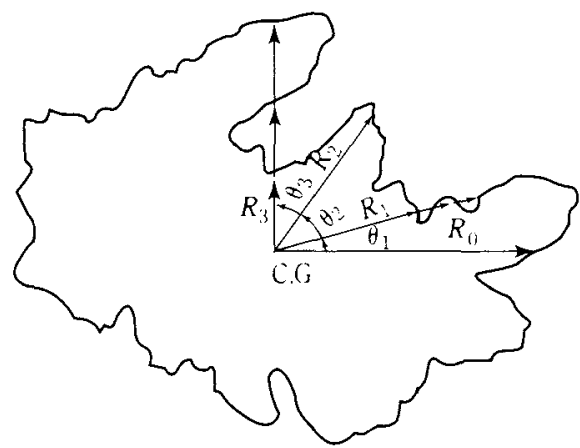

(b)

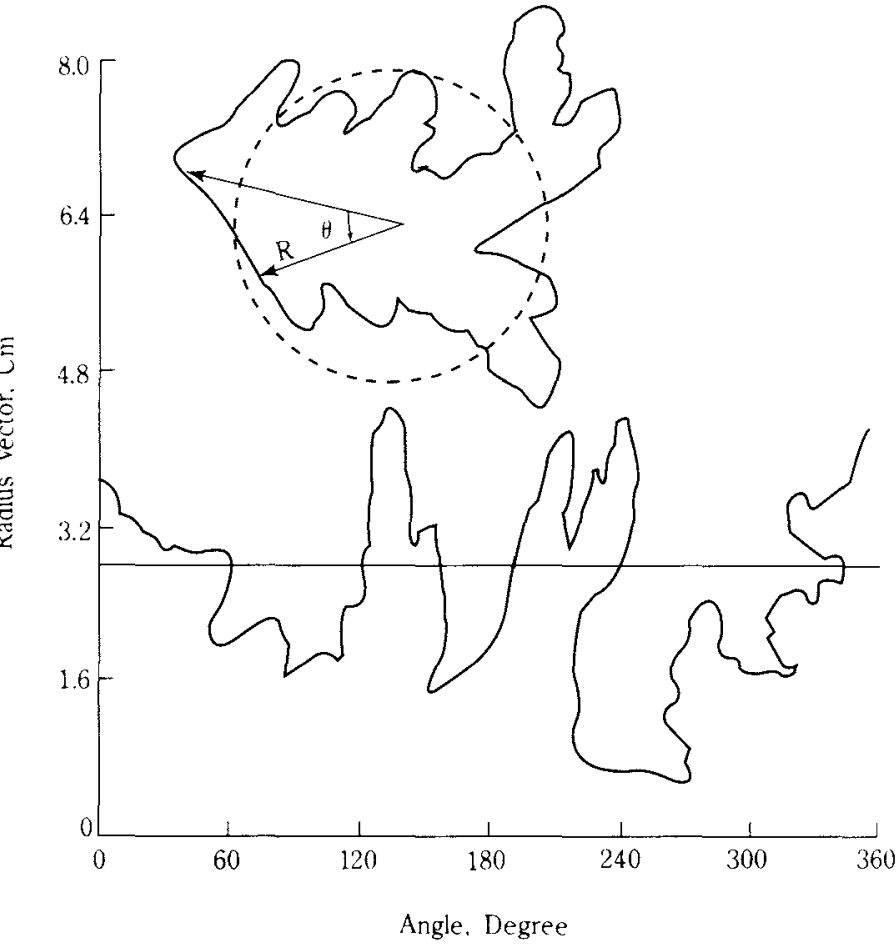

Fig. 5 (a) and (b) are two different ways of representing nonholomorphic or nonanalytic particle profile.

related to and inseparable from each other. Most of the literature in the area of powder characterization is clustered around the measurement of these basic intrinsic properties and characteristics derived thereof in one form or another. Various methods available in the literature for this purpose can be divided into four general classes ${ }^{5}$ : (i) the use of words to convey shape characteristics (verbalization of shape), (ii) the assessment of individual particle characteristics, (iii) shape generation by various methods and (iv) measurements of bulk properties of powders. Among these four classes, the (i) deals with the means of description, the (ii) and the (iv) deal with the means of observation and the (iii) deals with the transformation of particle profile into mathematical form. The succeeding sub-section describes the important methods lying under different general classes. 


\subsection{Werbalization of Shape}

Words like discs, blades, rods and equidimensionals have been used, based on the breadth to length and thickness to breadth ratios of the powder particles, to classify the powders into different shape groups. In Heywood's system ${ }^{6}$ of classification each particle is allocated to a different shape group which resembles one of the four geometrical forms - angular tetrahedral, angular prismoidal, subangular and rounded. The allocation of particles to a shape group is done based on the measurement of volume coefficient and surface coefficient. British standard ${ }^{7}$ gives a glossary of descriptive terms like acicular, angular, fibrous, irregular, nodular, crystalline, dendritic, flaky, granular and spherical for characterising powder particles. These conventional terminologies refer to two distinct characteristics of a powder particle namely, the geometrical form and the relative proportion of length, breadth and thickness. Riley and Magnuson ${ }^{8}$ characterized the refractory powder particles into four morphological classes - particles with hollows/ porosity in them, dense particles, agglomerated particles and hollow particles equivalent to agglomerated pores. Beddow ${ }^{9}$ has explored the possibility of using the concept of information theory to measure the information content of these linguistic variables (words or sentences) to characterize the powder particles. With the advent of fast character manipulating computers the concept can be used in pattern recognision technique to characterize powders.

\subsection{Assessment of individual particle charac- teristics}

Perhaps, the first attempt in quantitatively characterizing the powder particle would have been the measurements of its length, breadth and thickness. In a pioneer effort, Heywood ${ }^{6}$ defined thickness, breadth and length as the three dimensions of an irregular particle resting on a plane in the position of its greatest stability. He defined thickness (T) as the distance between two planes parallel to the planes of greatest stability and tangent to the surface of the particle, breadth (B) as the minimum distance between two parallel planes which are perpendicular to the planes defining thickness and are tangential to the opposite surfaces of the particles and length $(\mathrm{L})$ as the distance between two parallel planes which are perpendicular both to those planes defining thickness and breadth and which are also tangential to the opposite surfaces of the particle. Figure 6 shows the classical measures of thickness, breadth and length of a particle. Based on these measurements
Heywood defined flatness ratio $u$ and elongation ratio $v$ as follows:

Flatness Ratio, $u=\mathrm{B} / \mathrm{T}$

Elongation Ratio, $v=\mathrm{L} / \mathrm{B}$

Heywood related these two ratios to the ratio $d / w$ (diameter of the circle having the same area as the projection of the particle/sieve aperture) by the following formula:

$$
\frac{d}{W}=0.98\left(\frac{2 v u^{2}}{u^{2}+1}\right)^{1 / 2}
$$

Tabulation of values for different $u, v$ and $d / w$ ratios can help in characterizing the powder particles to a certain extent.

Davies $^{10}$ has measured length, breadth and thickness using Quantimet 720 Image Analyzer and utilized these information to construct triaxial controur diagrams of shape-sorted beach sands and silicon carbide powder. Hausnes ${ }^{11}$ compared the characteristics of the complicated shapes with the rectangles of minimum area (length a and breadth b) drawn around the projection of the particle in the most stable position, Figure 7. He defined three factors
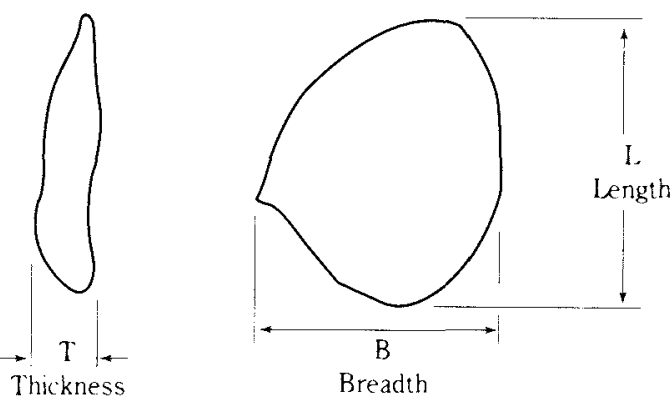

Fig. 6 Classical measures of thickness, breadth and length.

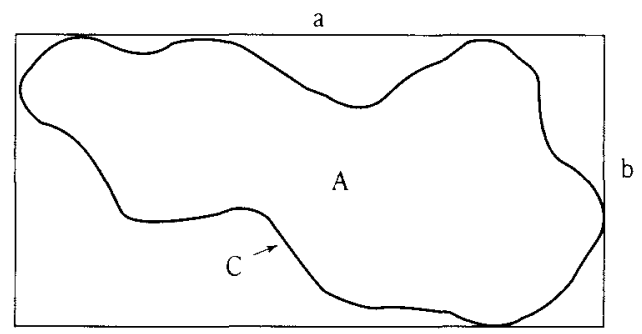

Fig. 7 Rectangle of maximum area drawn around the particle projection. 
as follows:

$$
\begin{aligned}
& \text { Elongation Factor }=a / b \\
& \text { Bulkiness Factor }=A /(a . b) \\
& \text { Surface Factor }=C^{2} /(4 \pi A)
\end{aligned}
$$

$C$ is the circumference of the projected particle and $A$ is the area of the projected particle.

Exner and Linck ${ }^{12}$ used a simple shape index $K$ which is defined as

$$
K=\frac{4 \pi A}{C^{2}}
$$

for characterizing the shape distribution of lead powders. The value of $K$ lies between 0 and 1 .

Comparison of surface area and volume of the particle with that of the equivalent sphere gives surface coefficient $f_{s}$ and volume coefficient $f_{v}$ as follows ${ }^{13}$ :

$$
\text { Surface area of the particle }=f_{s} \cdot \bar{d}^{2}
$$

Volume of the particle $=f_{v} \cdot \bar{d}^{3}$

$d$ is the projected average diameter of the particle.

To give an idea of the overall shape of the particle Wadell ${ }^{14}$ defined a term circularity as follows:

$$
\text { Circularity }=\frac{\begin{array}{c}
\text { circumference of a circle of the same } \\
\text { area as the particle }
\end{array}}{\text { circumference of the particle }}
$$

Sphericity which also conveys the idea of the overall shape was defined by Wadell ${ }^{15}$ as follows:

$$
\begin{aligned}
& \begin{array}{c}
\text { surface area of a sphere of the same } \\
\text { volume as the particle }
\end{array} \\
& \text { actual surface area of the particle }
\end{aligned}
$$

For two dimensional projection of particle,

$$
\text { Sphericity }=\frac{\begin{array}{c}
\text { diameter of a circle with the same } \\
\text { area as the particle projected on } \\
\text { the two dimensional plane }
\end{array}}{\begin{array}{c}
\text { diameter of the smallest circle } \\
\text { that circumscribes the } \\
\text { particle projection }
\end{array}}
$$

Circularity and sphericity are the measures of the degree to which the shape of a particle approaches a circle and a sphere, respectively. To take into account of the fine details of the particle Wadell ${ }^{15}$ defined roundness which is usually taken to be a measure of the sharpness of corners and edges of the particle, as follows:

$$
\text { Roundness }=\frac{\sum_{i=l}^{p} r_{i} / p}{R}
$$

$r_{i}$ are the radii of curvature of $p$ corners of a projection or section of the particle and $R$ is the maximum inscribed radius of the particle. Roundness value range from 0 to 1 .

Lees ${ }^{16}$ has shown that forms of identical roundness value can have visibly different actual roundness and hence, this led him to propose a measure of angularity of the particles in the following way, Figure 8 .

$$
\text { Angularity }=\sum_{i=1}^{3} \sum_{j=1}^{p}\left(180-\beta_{i j}\right) \frac{g_{i j}}{r_{i}}
$$

$\beta_{i j}$ is the angle of each corner and $g_{i j}$ is the distance of the corner from the centre of the maximum inscribed circle of radius $r_{i}$ for each of the three sections through the long, intermediate and short axes of the profile. Defined this way, angularity increases by increase in acuteness of the corners, increase in number of corners and increase in relative distance of corners from the centre of the particle and varies from 0 to $\infty$.

Using the concept of mechanics to describe the distribution of mass in a fine particle profile, Medalia ${ }^{17}$ and Medalia and Heckman ${ }^{18}$ calculated the dimensions of an ellipse having the same radii of gyration about the central principal axes as those which the fine particle profile would have if it were a thin lamina fine particle. Three shape factors for carbon black

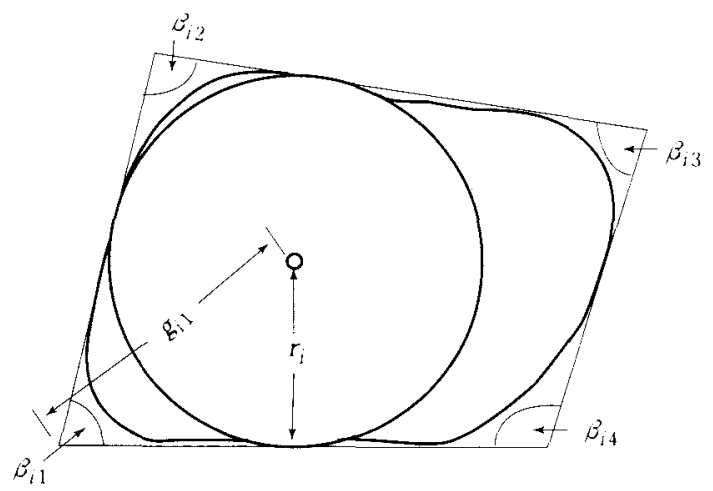

Fig. 8 2-D angularity of a particle. 
aggregates were then defined as follows, Figure 9.

$$
\begin{aligned}
& \text { Anisometry }=K_{A} / K_{B} \\
& \text { Bulkiness }=\frac{\text { area of ellipse }}{\text { area of sihouette }}=\frac{4 \pi K_{A} K_{B}}{A}
\end{aligned}
$$

$$
\text { Surface Factor }=(\text { Anisometry })(\text { Bulkiness })-1
$$

$A$ is the area of silhouette of particle, and $K_{A}$ and $K_{B}$ are the larger and smaller radii of gyration about the central principal axes of the radius-equivalent ellipse.

Medalia ${ }^{19}$ calculated three shape factors for both two and three dimensional bodies. Medalia's shape factors are based on the mechanics and therefore he referred to them as the dynamic shape factors. These shape factors are insensitive to highly reentrant and textured particles.

The shape factor, Shah, which is the ratio of the number of downward pointing projections on a small set of particles to the total particle population of the sample was developed by Staniforth and Rees ${ }^{20}$ specifically to quantify the re-entrant particles. Shah is most useful in describing the mean particle shape of a sample of bulk powder but it can also be calculated for individual powder particle. The value of Shah for re-entrant particles increases to a maximum which is governed by the resolution at each magnification. Shah quantifies the degree of re-entrancy of

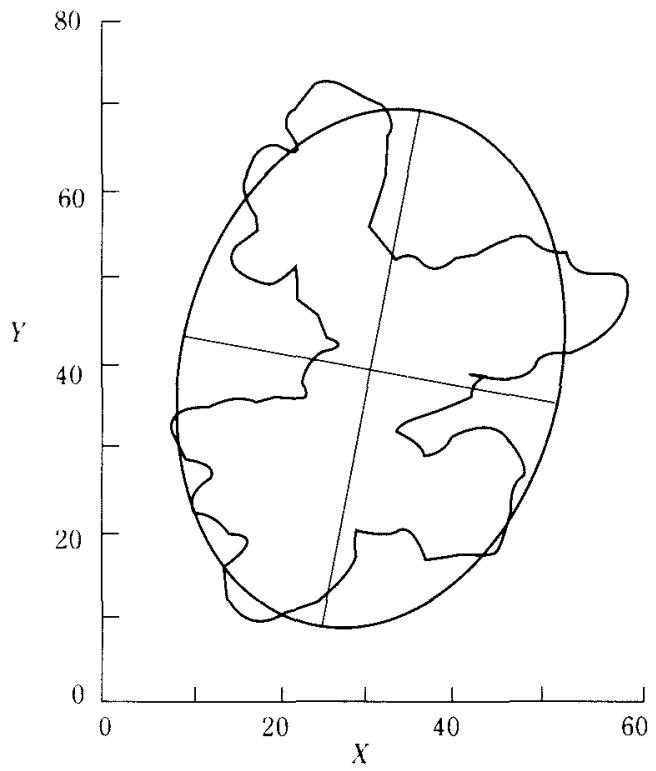

Fig. 9 Silhouette of carbon black aggregate with central principal axes and radius-equivalent ellipse. a particle but it is unable to characterize its geometric form. Staniforth and Rees ${ }^{21}$ combined Shah and Heywood shape factor $\left(f_{v} / f_{s}\right)$ and formed a two dimensional matrix which is capable of describing re-entrant and non-re-entrant, simple and complex geometric forms.

Danieisson 22 proposed a shape factor $G$ which is based on average distance between a picture element and its nearest border point $\bar{g}$ and defined $G$ by the following formula:

$$
G=\frac{A}{9 \pi(\bar{g})^{2}}
$$

$A$ is the area of the particle profile. Fast computation of $G$ requires that the particle be degitized into several pixels. Degitized version of the formula is given by

$$
G=-\frac{M^{3}}{9 \pi\left(\sum_{i=1}^{M} g_{i}\right)^{2}}
$$

$M$ is the total number of pixels in the profile and $g_{i}$ is the distance of the ith pixel within the profile from its nearest border point. It is claimed by Danielsson that $G$ captures the compactness of the profile.

Kaye $^{23}$ emphasizes that the quantitative gross shape descriptors of particle profiles should not tend to infinity as the scale of scrutiny is increased, and should be relatively easy to measure and for that he defined, external compactness as follows:

$$
\begin{aligned}
& \text { External Compactness }= \\
& \qquad \begin{array}{l}
\left(\frac{\text { diameter of a circle of equal area }}{\text { diameter of embracing circle }}\right)^{2} \\
=\left(\frac{D_{A}}{D_{E m}}\right)^{2}
\end{array}
\end{aligned}
$$

Since it is possible to have the same external compactness but different general shapes of the profiles, Kaye uses another index chunkiness in conjunction with the former.He defines chunkiness as follows:

$$
\text { Chunkiness }=B_{m} / L_{m}
$$

$L_{m}$ is the maximum length of the profile and $B_{m}$ is the maximum breadth of the projection of the profile on a direction at right angle to that in which $L$ is measured. Figure $\mathbf{1 0}$ shows the basic measurement 


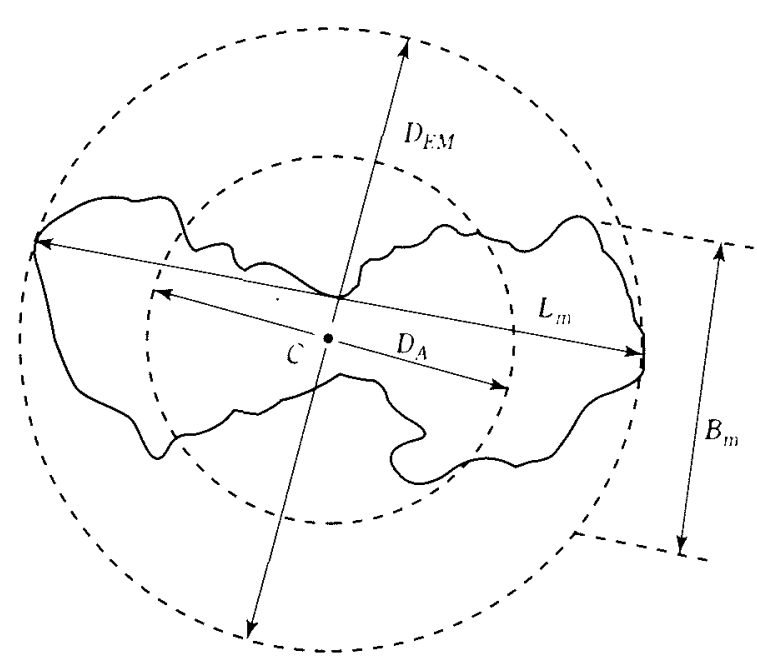

Fig. 10 Chunkiness and external compactness of the particle profile.

used for calculation. By plotting the external compactness and chunkiness in two dimensional data space Kaye expresses the variability of shape exhibited by the atomized copper-lead powder and sponge iron powder. Since it is possible to completely separate the two powders in three dimensional data space, Kaye $^{24}$ expressed a measure of the ruggedness of the particle as follows:

$$
-\delta=(D-1)
$$

$D$ is the ruggedness index or fractal dimension having the value between 1 and 2 and $\delta$ is the slope of perimeter-random walk stride graph as shown in Figure 11.

To describe the structure of a two dimensional particle profile, concept of geometric signature waveform was developed by Schwarcz and Shane ${ }^{25}$. They used the magnitude of the vector $R$ which rotates with constant angular velocity about the pivot point to generate the waveform by plotting $R$ against $\theta$, Figure 12. The centre of the smallest circumscribing circle was used as the pivot point. The magnitude of the vector is normalized with respect to the largest value generated as the vector rotates about the pivot point touching the periphery of the profile. This waveform is vulnerable to variations in the location of the pivot point as shown in Figure 13. Reentrant particle profiles cannot be described by geometric signature waveform for the simple reason that rotating vector acquires multiple values as it crosses a re-entrant loop of the profile.

Kaye $^{26}$ generated Feret's diameter signature waveform (also called FERETS waveform) by plotting the magnitude of the Feret's diameter of the profile against the angle of orientation with respect to a fixed direction as the profile is rotated with uniform angular motion, Figure 14. Normalization of data can be done by dividing the measured projected
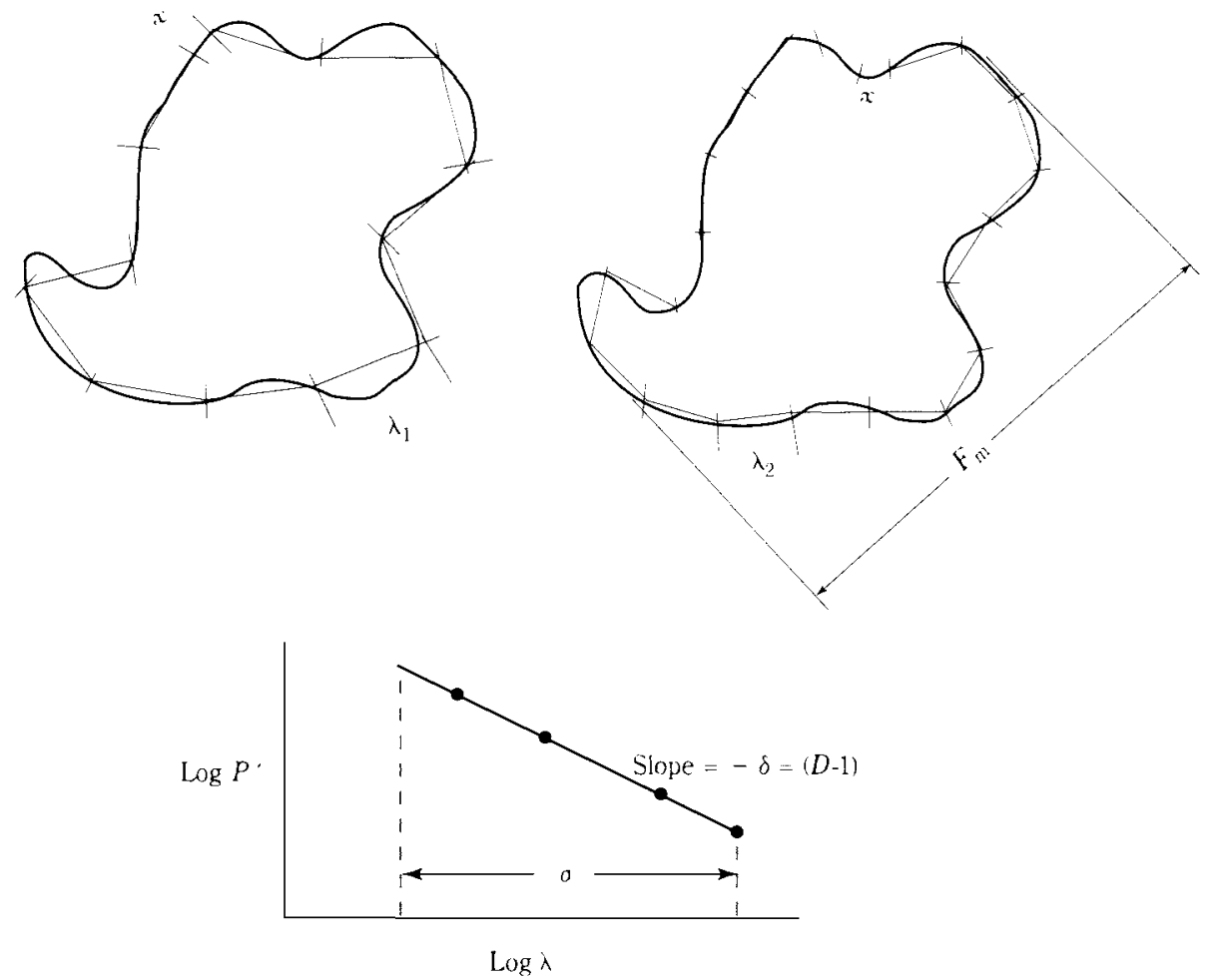

Fig. 11 Perimeter-random walk stride graph. 
Fig. 14

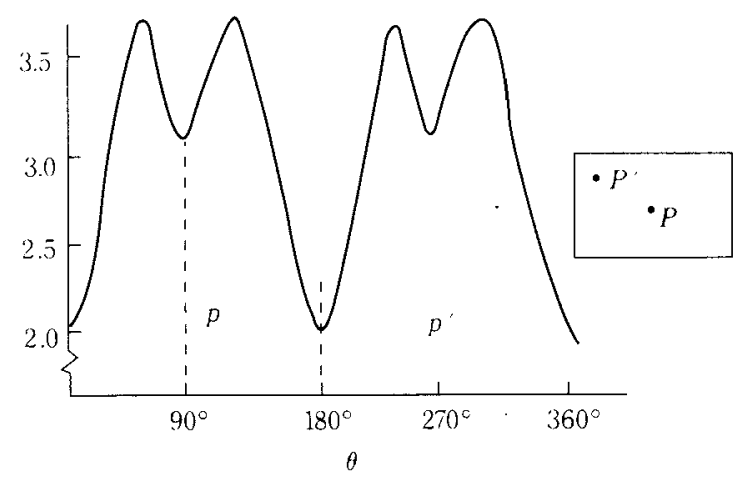

Fig. 14 Feret's diameter signature wafeform showing the independence of the point of rotation.

dependent of the point about which the profile is rotated and is related to the perimeter of the profile.

Cauchy signature waveform ${ }^{27}$ consists of the magnitude of the projected area plotted against the angular position rotated through $90^{\circ}$ about the mounting axis and a further $90^{\circ}$ perpendicular to the axis, Figure 15. Measurement of any concavities on the surface of the structure is not possible by this procedure.

Feret ${ }^{28}$ suggested that the average value of the projected length of an array of particles with reference to a given direction can be used to characterize that array of the particles. Thus, originally it was used as a statistical diameter. However, the common usage has extended the term Feret's diameter to describe the projected dimension of an individual profile. Feret's diameter is the distance between the tangents which touch the two extremities of the particle profile in its position of rest at right angle to the direction of scan, Figure 16. Similarly, Martin's diameter is the distance which divides the particle profile into two equal halves in the direction of scan when the particle is in a position of rest, Figure 17. These direction vary depending on the orientation of the particle profile. Ratio of Martin's diameter to Feret's diameter of the particle population has been suggested as a useful shape factor, however, Church ${ }^{29}$ has analyzed the major problem associated with this ratio as a shape factor. He has indicated that useful mathematical relationship can be set up between expected values of Martin's diameter and Feret's diameter and various shape factors for elliptical particles and also for particles of other shapes can be obtained.

Stereology which studies the three dimensional structure of materials from two dimensional sections or projections is being used for powder characteriza-

meter for a given profile by the maximum Feret's diameter for that profile. FERETS waveform is in 


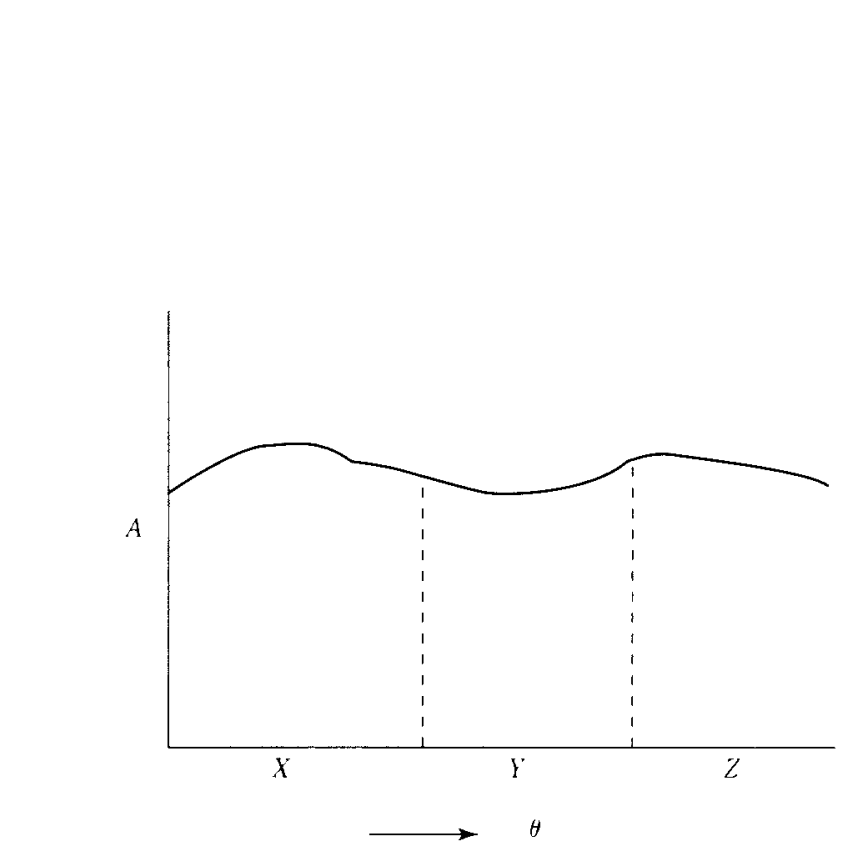

Fig. 15 Cauchy signature waveform generated by plotting the area of the silhouette against the angle of rotation about $x, y$ and $z$ of the fine particle.

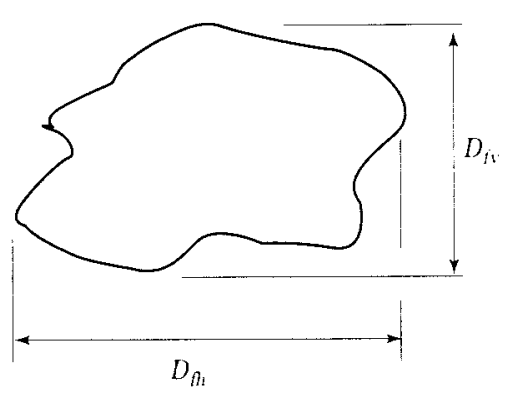

Fig. 16 Horizontal and vertical Feret's diameter.

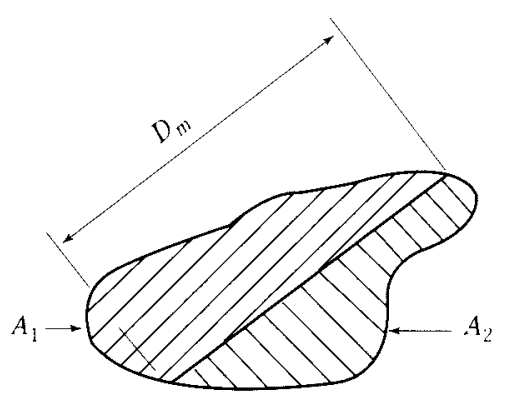

$A_{1}=A_{2}$

Fig. 17 Martin's diameter.

tion. Underwood ${ }^{30}$ has discussed the types of measurements obtained from sections and projections of the powder particle and the applicable formulae that serve to related particle characteristics or behaviour. Beddow et al. ${ }^{31}$ selected few such measurements to define dimensionless features and subsequently applied for classifying four different types of powder by use of a pattern recognition technique. Underwood ${ }^{32}$ has listed many shape indices which are evaluated by using stereological methods. Singh and
Ramakrishnan ${ }^{33-35}$ used such stereological shape indices and established their correlation with the bulk properties of the powder and subsequently used it for characterizing copper powders of different origins.

There are host of parameters of similar nature ${ }^{36,37}$, but only few selected ones have been presented here to understand the general trend and utility of such parameters.

\subsection{Shape generation and the recent develop- ments}

Presently available shape generation methods have been put under four categories ${ }^{5}$ : Matrix mapping, polynomial fitting, syntactic methods and Fourier analysis technique. In matrix mapping, the silhouette of the particle is scanned by photoscanner or some such device which divides it into different grey levels. The particle shilhouette is thus converted into digital map which becomes amenable for further computer processing. Polynomial generation of particle profile is simply a curve fitting task where different degree of polynomials are tried for representing the particle profile as closely as possible. There is a limit to attain the closeness of fit after which wide deviation starts when the degree of polynomial is increased. In syntactic method ${ }^{38}$, the specific pattern is developed from a given set of pattern primitives by the operation of rules of the grammar of the language. Beddow et al. ${ }^{39}$ have used picture descriptive language (PDL) grammar to explain the construction of a pentagon.

In Fourier analysis technique of shape generation, the particle profile is analyzed to yield large number of Fourier coefficients. These coefficients capture all the details of the profile. By reversing the process, i.e., by using synthesis technique the profile can be regenerated to its near original shape.Schwardz and Shane ${ }^{25}$ used Fourier analysis technique to measure the shape characteristics of sand grains. Ehrlich and Weinberg ${ }^{40}$ used this technique to reproduce profiles of quartz grains very precisely and also to characterize grains from different sources. Several researches ${ }^{41-49}$ have used this technique to characterize powder particles. Eppler and Meloy ${ }^{50}$ have chronicled the development of this technique and have shown its potential applicability to a host of engineering and industrial problems. Fourier analysis technique has been explored extensively by Beddow and co-researchers ${ }^{50-60}$ and this has led to identify three different methods of Fourier analysis depending on the nature of the particle profile and on the information required for characterization. These three different methods are $(R, \theta)$ method, $(\phi, \ell)$ method 


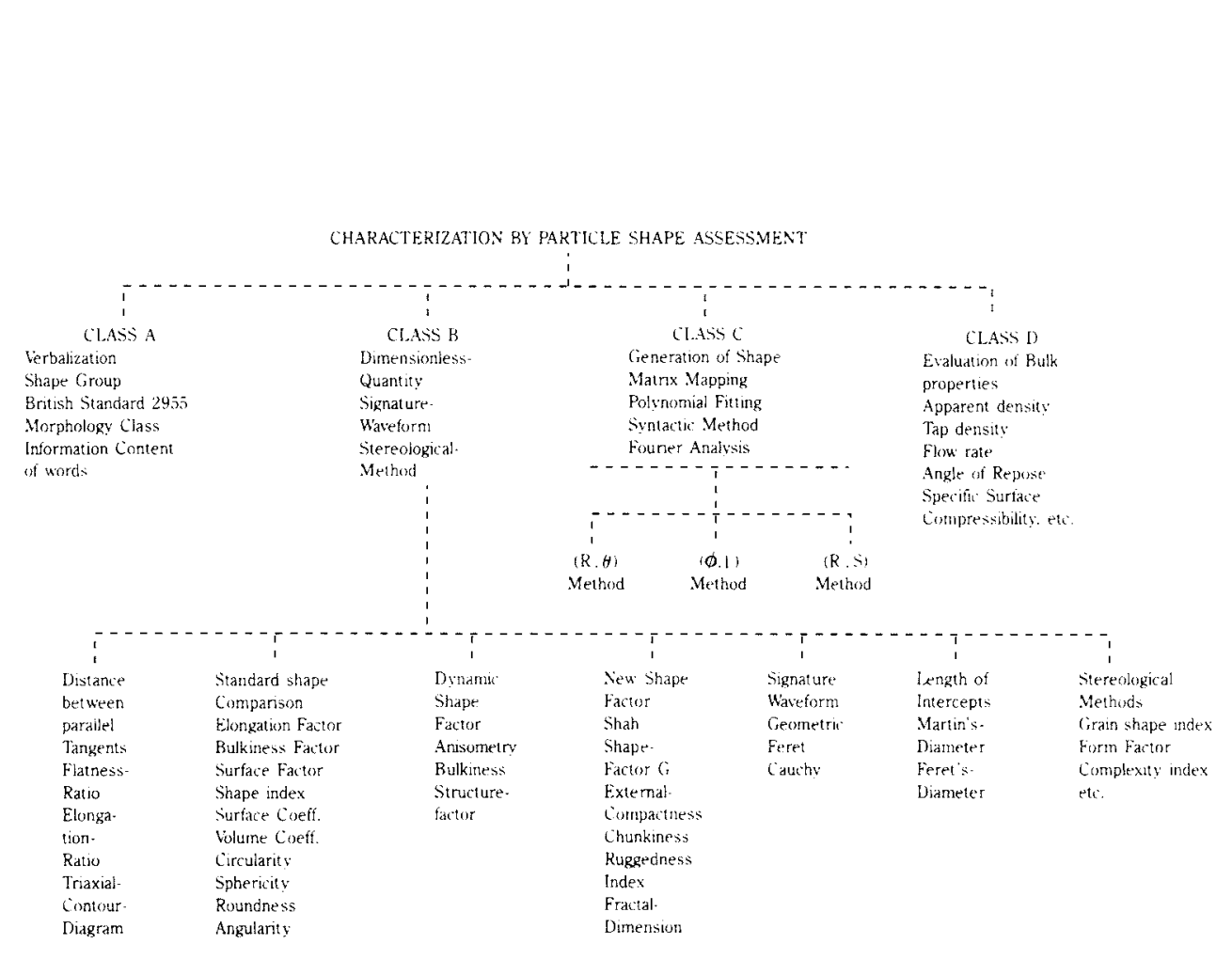

Fig. 18 Classification of characterizing parameters based on particle shape assessment.

and $(R, S)$ method.

In $(R, \theta)$ method $^{40,54,59}$, the particle profile is degitized and a set of $\left(x^{\prime}, y^{\prime}\right)$ coordinates are obtained. This is then transformed to a set of $(x, y)$ coordinates with reference to the centre of gravity. These $(x, y)$ coordinates are then transformed to polar representation $(r, \theta)$ and then $R$ is represented as a function of $\theta$ by truncated Fourier series:

$$
R(\theta)=a_{o}+\sum_{n=1}^{N}\left(a_{n} \operatorname{Cos} \mathrm{n} \theta+b_{m} \operatorname{Sin} \mathrm{n} \theta\right)
$$

where,

$$
\begin{aligned}
& a_{o}=\frac{1}{2 \pi} \int_{0}^{2 \pi} R(\theta) d \theta \\
& a_{n}=\frac{1}{\pi} \int_{0}^{2 \pi} R(\theta) \cos n \theta d \theta \\
& b_{n}=\frac{1}{\pi} \int_{0}^{2 \pi} R(\theta) \operatorname{Sin} n \theta d \theta \\
& n=1,2,3, \ldots \ldots \ldots \ldots . . .
\end{aligned}
$$

$a_{o}, a_{n}$ and $b_{n}$ are zeroth order and nth order Fourier coefficients containing size and shape information. The Fourier coefficients $\left\{a_{n}, b_{n}\right\}$ contain all the informations about the particle profile to reconstruct the particle profile analytically by equation (23). Since the Fourier coefficients and phase angle are not invariant to coordinate transformation, the particle shape representation is not unique.
The Fourier coefficients are transformed to the set of invariant features - size, shape, roughness, microroughness, partial rotational symmetry, partial mirror symmetry, partial inversion symmetry and moments of the radial distribution - according to the theory of morphology 61,62 . These features measure well-defined properties of the particle profile.

The $(R, \theta)$ method in its classical form cannot handle multivalues of $R$ at a given value of $\theta$. This is a significant drawback of the classical $(R, \theta)$ method. However, using the variational method other boundary representations for reentrant profiles are possible by using a generalized concept of the angle and this gives to the generalized $(R, \theta)$ method for the reentrant particle profile ${ }^{62}$.

In $(\phi, \ell)$ method $^{46,48,55,63}$, the particle profile is parameterized by a normalized arc length and a change of slope from its starting point. The normalized function can be written as

$$
\phi^{*}(t)=\mu_{0}+\sum_{n=1}^{N}\left(a_{n} \operatorname{Cos} n t+b_{n} \operatorname{Sin} n t\right)
$$

where,

$$
\begin{aligned}
& t=\frac{\ell}{L} 2 \pi ; t=(0,2 \pi) \text { for } \ell=(0, L) \\
& \ell_{i}=\sum_{i=1}^{K} \sqrt{\Delta x_{i}^{2}+\Delta y_{i}^{2}}
\end{aligned}
$$

$K=$ arc length from starting vertex to the $K$ th vertex $=1,2,3 \ldots 2 N+1$, the number of coordinate points on profile 
$\stackrel{N}{L}=\sum_{i=1}^{K} \ell_{i}$

The total arc length is $L$ and the normalized parameter is t. $a_{n}$ and $b_{n}$ are the Fourier coefficients.

This method has the advantage that is can analyze both holomorphic and nonholomorphic particle profiles. This method does not yield analytical expression for the regenerated $(x, y)$ coordinates. Regenerated coordinates must be calculated using numerical techniques and hence, important geometrical and physical quantities, such as area, moment of inertia, symmetry, etc. cannot be evaluated analytically.

In $(R, S)$ method $^{60,62},(x, y)$ coordinates on the particle profile are transformed to polar coordinate system $(\gamma, \theta)$. A normalized arc length $\mathrm{s}$ is calculated as in $(\phi, \ell)$ method. Since $\gamma$ as a function of $s$ is a function of period $2 \pi$, a Fourier series may be used to represent $R(s)$. In addition, $\theta$ as well $\theta^{\prime}$ as functions of $s$ are also functions of period $2 \pi$ and may also be represented by Fourier series. Thus, $R(s)$ and $\theta^{\prime}(s)$ are represented as functions of normalized arc lengths, as follows:

$$
\begin{aligned}
& R(s)=a_{0}+\sum_{n=1}^{N}\left(a_{n} \operatorname{Cos} n s+b_{n} \operatorname{Sin} n s\right) \\
& \theta^{\prime}(s)=C_{0}+\sum_{n=1}^{N}\left(C_{n} \operatorname{Cos} n s+D_{n} \operatorname{Sin} n s\right)
\end{aligned}
$$

where,

$$
\begin{aligned}
& s=\frac{\ell}{L} 2 \pi \\
& \ell_{i}=\sum_{i=1}^{K} \sqrt{\Delta x_{i}^{2}+\Delta y_{i}^{2}} \\
& L=\sum_{i=1}^{K} \ell_{i}
\end{aligned}
$$

and

$$
\theta^{\prime}(s)=\frac{d}{d_{s}} \quad[\theta(s)]
$$

Both reentrant and nonreentrant particle profiles can be analyzed by this method. It can also be used to differentiate between reentrant and nonreentrant profiles since profile containing reentrant points will have negative values of $\theta^{\prime}(s)$ at certain points of reentrance, whereas profiles which do not contain reentrant points will have positive values of $\theta(s)$ for all s. Analytical calculation of important geometrical and statistical quantities such as area, moment of inertia, symmetry, moments of radial distribution, etc. is possible by this method. A major disadvantage of this method is that it requires two Fourier expansions. This reduces the efficiency of this method as compared with $(R, \theta)$ and $(\phi, \ell)$ methods.

Since, even the minor differences in the shape of the particle is reflected in the Fourier coefficients, each particle can be uniquely distinguished from the other particles by simply comparing their harmonic amplitudes. However, a discriminatory ability which forces us to distinguish among all the particles encountered is not required. Such a level of differentiation would yield an almost infinite variety, which would be useless ${ }^{64}$. Therefore, if one wants to classify one powder lot from another, one must take into account the overall morphological features for each powder and make a comparison. Thus, to make use of the Fourier coefficients as characterizing tool, further treatment of the data becomes imperative for which many techniques have been developed and used by many researchers ${ }^{2,46,47,49,61,65-68}$.

Walsh Transform has been used by Lew et al. 69 for morphological classification of powders. It has been pointed out that a circular shift in starting point produces a different set of Walsh coefficients. This clearly indicates that Walsh transform in unsuitable for morphological analysis. Fast Fourier Transform (FFT) has seen used to particle silhouettes to obtain morphological data ${ }^{70,71}$. Beddow ${ }^{72}$ mentions the use of Ramtek device in particle image analysing system to analyze the color of particles as a morphic feature which assists in differentiation and classification processes. Use of color as a morphic feature extraction is one of the upcoming developments in the area of powder characterization.

\subsection{Bulk properties evaluation}

For a mass of powder, the bulk properties, such as apparent density, tap density, flow rate, angle of repose, specific surface, compressibility, etc. are dependent to a large extent on size, size distribution, shape and shape distribution of the powder particles and hence, these bulk properties have served as important parameters for characterizing powder. Many of these bulk properties can be determined by standard test procedures and the other can be evaluated by developing appropriate techniques.

Hausner ${ }^{11}$ used the ratio of tap density to apparent 
density as a qualitative measure of friction in the moving powder mass to distinguish three differently shaped copper powders of similar size distribution. This ratio, called friction index, was found to be high for flaky powder and low for spherical powder. Tap density to apparent density ratio has been applied as a shape parameter to classify electrolytic, reduced and atomized copper powders ${ }^{3,73}$. Kostelnik et al. ${ }^{74}$ have shown the influence of this ratio on the compaction behaviour of copper and iron powders. Riley and Mann ${ }^{75}$ using glass particles of four different shapes and Singh ${ }^{3}$ using copper powders of different origins have shown that angle of repose increases with increasing departure from sphericity. Use of flow rate and specific surface as characterizing parameters are not hard to explore. Density-pressure relationship curves which can be obtained by compacting the powder successively at different pressures can be made use of in characterizing powders of different origins ${ }^{76}$. For successful characterization of powders by bulk properties evaluation it is essential that the measurements by carried out under identical conditions since these properties are sensitive to experimental conditions.

\section{Critical assessment}

The parameters for characterizing the powders by particle shape evaluation are either of qualitative or quantitative nature. Words like acicular, angular, fibrous, etc. which are descriptive are qualitative parameters and different meanings can be ascribed to them by different persons.'Thus, ambiguity creeps in while attempting to communicate the characteristics of powders using these parameters and therefore, such type of qualitative parameters are of little value in the scientific community. On the other hand, many of the quantitative parameters are based on the gross shape of the powder particles and therefore, finer details of particle shape are not taken into account by these parameters. Many of such parameters can have same values for differently shaped powders and therefore, misclassification can result when these parameters are used for powder characterization. The shape factor Shah by Staniforth 20 is a function of magnification and as the magnification increases, the value of Shah increases. This type of parameter cannot be unequivocally selected for characterizing the powder. Parameters like external compactness and chunkiness defined by Kaye ${ }^{23}$ do not take into consideration the fine-scale perturbations of the particle profile. The use of signature waveforms is yet to be explored. Stereological methods can be applied both to indivi- dual particle or systems of many particles. Since these methods are statistical in nature, large number of measurements has to be made to achieve high degree of accuracy and therefore, automatic image analysis systems have to be used to accomplish the task.

One of the major drawbacks of all such parameters is that in course of condensing the informations for characterizing the powders, one loses the sight of the actual particle profile and it becomes well-nigh impossible to reconstruct the original powder particle profile from these parameters. But, in the case of Fourier analysis technique it can be seen that it is very much possible to reconstruct the particle profile from Fourier coefficient to its near original shape and hence, this technique holds a great promise as a characterizing tool. $(\phi, \ell)$ method and modified $(R$, $\theta)$ method which take into account both holomorphic and nonholomorphic particle profile have been used extensively while $(R, S)$ method which involves two Fourier series expansions has found limited application because of the difficulty arising in handling the huge quantity of data generated by this method.

Bulk properties are sensitive to particle morphology and also to experimental conditions. Hence, if the bulk properties are to be used for characterization of powders, experimental conditions have to be strictly controlled, otherwise, variation in bulk properties cannot totally be attributed to the change in particle characteristics.

Thus, it can be inferred that presently no single method exists which can characterize the powder completely and therefore, one has to use combination of different methods for characterizing the powder, but even then, the characterization remains short of mark as far as the finer aspects of particle characteristics are concerned; nevertheless, judicious combination of different methods can solve the problem to a great extent.

\section{References}

1) Hausner, H.H., Environmental effects on the behaviour of metal powder. Int. J. Powder Met. Powder Tech., 14 (1978) 323.

2) Meloy, T.P., A hypothesis for morphological characterization of particle shape and physiochemical properties. Powder Tech., 16 (1977) 233.

3) Singh, Paramanand., Characterization of Powders Ph.D. Thesis, IIT Bombay, India, 1983.

4) Barrett, P.J., The shape of rock particles, a critical review. Sedimentology, 27 (1980) 291.

5) Beddow, J.K., Sisson, K. and Vetler, A.F., Particle shape analysis, Part I. Powder Met. Int. 8 (1976) 69. 
6N Heywood, H., The Scope of particle size analysis and standardization. In proceedings of Symposium on Particle size analysis, suppliment to Trans. Inst. Chem. Engr., 25 (1947) 14.

7) Clossary of terms relating to powders. British Standard 2955 (1958)

8) Riley, R.E. and Magnuson, J.E., As cited in Ref. 4.

9) Beddow, J.K., Particle morphological analysis. In Advanced Particulate Morphology, eds. Beddow, J.K. and Meloy, T.P. CRC Press, Boca Raton, Florida, (1980) 1-84.

10) Davies, R., A simple feature-space representation of particle shape.Powder Tech., 12 (1975) 111.

11) Hausner, H.H., Latest development in the characterization of powders. In Powder Metallurgy and Material Strengthening, eds. Dhar, P.R., Sen, P.K., Basu, S. and Mukunda, P.G. Oxford and IBH Publishing Co., Calcuatta, (1970) 9-18.

12) Exner, H.E. and Linck, E., Image analysis for characterization of size-and shape distribution of lead powders. Powder Met. Int. 9 (1977) 131.

13) Orr, C.J. and Dallavalle, J.M., Fine Particle Measurement, Macmillan Company, NY (1959) 25-26.

14) Wadell, H., Sphericity and roundness of rock particles.J. Geol, 41 (1993) 310.

15) Wadell, H., Volume, shape and roundness of quartz particles. J. Goel, 43 (1935) 250.

16) Lees, G., A new method for determining the angularity of particles. Seimentology, 3 (1964) 2 .

17) Medalia, A.I., Morphology of aggregates: Calculation of shape and bulkiness factor; Application to computersimulated random flocks. J. Colloid Interf. Sci., 24 (1967) 393.

18) Medalia, A.I. and Heckman, F.A., Morphology of aggregates: 2. Size and shape factors of carbon block aggregates from electron microscopy, Carbon, 7 (1969) 567.

19) Medalia, A.I., Dynamic shape factors of particles. Powder Tech., 4 (1970/71) 117.

20) Staniforth, J.N. and Rees, J.E., Shape classification of re-entrant particles I: The Shape factor Shah. Powder Tech., 28 (1981) 3.

21) Staniforth, J.N. and Rees, J.E., Shape classification of re-entrant particles II: Description of re-entrant and non re-entrant particle shapes. Powder Tech., 28 (1981) 9.

22) Danielsson, Per-Erik., A new shape factor. Computer graphics and Image Processing, 7 (1978) 292.

23) Kaye, B.H., Fractal and shape index description of the shape and surface structure of metal powders. In Modern developments in Powder Metallurgy, eds. Hausner, H.H., vol.12, 1981, pp 67-82.

24) Kaye, B.H., Specification of the ruggedness and/or texture of a fine particle profile by its fractal dimension. Powder Tech., 21 (1978) 1.

25) Schwarcz, H.P. and Shane, K.C., Measurement of particle shape by Fourier analysis. Sedimentology, 13
(1969) 213.

26) Kaye, B.H., The use of Feret's diameter signature waveform as a shape characterization parameter. In Fine Particle Science. Proceeding of the symposium on the characterization of large colloidal particles at Anahelm, California, 1978.

27) Kaye, B.H., Sinha, S.N. and Lablanc, J.E., Signature waveforms for describing the three dimensional morphology of fine particles. Proceedings of Int. Symposium on Recent advances in particulate science and technology, IIT Madras, Preprints, Vol. 1, 1982, A-11.

28) Feret, R.L., as cited in Ref. 26.

29) Church, T., Problems associated with the use $f$ the ratio of Martin's diameter to Feret's diameter as a profile shape factor. Powder Tech., 2 (1968/69) 27.

30) Underwood, E.E., Stereological analysis of particle characteristics. In Testing and Characterization of Powders and Fine Particles. eds. Beddow, J.K. and Meloy, T.P., Heyden \& Sons Ltd.,1980, pp 77-96.

31) Beddow, J.K., Lew, G. and Vetter, A.F., On the classification of four different types of powder by a pattern recognition technique applied to particle shape characteristics. Report No. G178-77 001, Materials Engineering Division, The University of Iowa, Iowa City, 52242, USA.

32) Underwood, E.E., Quantitative Stereology, AddisonWesley, Reading, Masschusetts, 1970, pp 227-232.

33) Singh, P.N. and Ramakrishnan, P., Correlation of shape indices with bulk properties of powders. Trans. of the PMAI, 7 (1980) 62-69.

34) Singh, P.N. and Ramakrishnan, P., Study of powder bulk properties in the light of stereologically evaluated particle characteristics. J. of Powder and bulk Solids Tech., 11 (1987) 13-17.

35) Balasubramaniam, V., Singh, P.N. and Ramakrishnan, P., Effects of some particle characteristics on the bulk properties of powders. Powder Met. Int., Vol. 16, 2 (1984) 56-59.

36) Pahl, M.H., Schadel, G. and Rumpf, R., Zusammenstellung von Teilchenformbeschreibungsmethoden. In Newsletter ' 76 in Stereology, ed. Ondracek, G., Gesellschaft fur Kernforschung mbH, Karlsruhe, 1976 , pp 89-107.

37) Barrett, P.J., The shape of rock profiles, a critical review. Sedimentology, 27 (1980) 291.

38) Tou, J.T. and Gonzales, R.C., Pattern Recognition Principles, Reading, Addison Wesley, 1974.

39) Beddow, J.K., Philip, G.C. and Nasta, M.D., On the use of pattern recognition methods in particle shape analysis. Planseeber Pulvermetall., 24 (1976) 167.

40) Ehrlich, R. and Weinberg, B., An exact method for characterization of grain shape.J. Sed. Petrol,, 40 (1970) 205

41) Czarnecka, E.T., and Gillott, J.E., A modified Fourier method of shape and surface texture analysis of planar sections of particles. J. Testing and Evaluation, 5 (1977) 292. 
42) Gearnecka, E.T., and Gillott, J.E., The effect of orientation on the analysis of shape and texture of concrete aggregates by modified Fourier method. J. Testing and Evaluation, 5 (1977) 299.

43) Czarnecka, E.T. and Gillott, J.E., Roughness of limestone and quartzite pebbles by the modified Fourier method. J. Sed. Petrol, 50 (1980) 587.

44) Gotoh, K., Representation of the size and shape of a single particle.Powder Tech., 12 (1975) 125.

45) Gotoh, K., Shape characterization of two dimensional forms. Powder Tech., 23 (1979) 131.

46) Singh, Paramanand. and Ramakrishnan, P., Morphological classification by harmonic analysis. Powder Met. Int., Vol. 20, 4 (1988) 12-15.

47) Singh, Paramanand, and Ramakrishnan, P., Morphological classification of copper powders of different origins by Fourier analysis. Powder Met., Vol. 31, 4 (1988) 287-292.

48) Paramanand. and Ramakrishnan, P., Regeneration of form by Fourier technique and its application in powder characterization. Part. Part. Syst, Charact. 5 (1988) 179-185.

49) Singh, Paramanand. and Ramakrishnan. P., Application of few salient strategies for reduction of Fourier analysis data for powder characterization. In Proceedings of international conference on Advances in Materials and Processes, ed. Ramakrishnan, Oxford \& IBH Publishing Co, 1993, pp 159-167.

50) Eppler, D.T. and Meloy, T.P., Applicability of sophisticated shape analysis technique to engineering and industrial problems-Fourier shape analysis. In Fine Particle Processing, ed. Somasundaram, P., Vol. 1, American Institute of Mining, Metallurgical and Petroleum Engineers, New York, 1980, pp 462-480.

51) Beddow, J.K. and Philip, G.C., On the use of a Fourier analysis technique for describing the shape of individual particles. Planseeber Pulvermetall, 23 (1975) 3.

52) Beddow, J.K. Philip, G.C. and Vetter, A.F., On relating some particle profile characteristics to the profile Fourier coefficients. Powder Tech., 18 (1977) 19.

53) Beddow, J.K. and Vetter, A.F., A note on the use of classifiers in morphological analysis of particulates. J. Powder and Bulk Solids Tech., 1 (1977) 42.

54) Beddow, J.K., Two different methods of morphological analysis of fine particles. Report No. G178 78 07, Materials Engineering Division, The University of Iowa, Iowa City, 52242, USA.

55) Fong, S-T., Beddow, J.K. and Vetter, A.F., A refined method of particle shape representation. Powder Tech., 32 (1979) 17.

56) Beddow, J.K., Fong, S-T., and Vetter, A.F., Morphological analysis of metallic wear debris. Wear, 58 (1980) 201.

57) Lee, Y., Beddow, J.K. and Vetter, A.F., Morphological analysis of fine particle mixtures. Powder Tech., 25 (1980) 137.
58) Beddow, J.K., Nasta, M.D. and Philip, G.C., Characteristics of particle signatures. Report No. NSF-76006, Materials Engineering Division, The University of Iowa, lowa City, 52242, U.S.A.

59) Luerkens, D.W., Beddow, J.K. and Vetter, A.F., Morphological Fourier descriptors. Powder Tech., 31 (1982) 209

60) Luerkens, D.W., Beddow, J.K. and Vetter, A.F., A generalized method of morphological analysis. Powder Tech., 31 (1982) 217.

61) Beddow, J.K. and Luerkens, D.W., Effects of powder production and of material processing on powder particle morphology of adipic acid. Powder Tech., 68 (1991) 163-173.

62) Luerkens, D.W., Theory and Application of Morphological Analysis, CRC Press, Boca raten, 1991, chapter 2,6 and 7 .

63) Singh, Paramanand. and Ramakrishnan, P., Particle shape quartization - a novel approach. Trans. of PMAI, 10 (1983) 19-26.

64) Beddow, J.K., Particulate Science and Technology, Chemical Publishing Co., New York, 1980, p 11.

65) Ehrlich, R., Brown, J., Yarus, J.M. and Eppler, D.T., Analysis of particle morphology data. In Advanced Particulate Morphology, eds. Beddow, J.K. and Meloy, T.P., CRC Press, Boca Raton, 1980, pp 101-119.

66) Beddow, J.K., Nasta, M.D., and Philip, G.C., Characteristics of particle signatures. In Testing and Characterization of Powders and Fine Particles, eds. Beddow, J.K. and Meloy, T.P., Heyden \& Sons Ltd., Florida, 1980, pp 44-55.

67) Singh, Pramanand., and Ramakrishnan, P., Powder signature - a strategy for powder characterization by Fourier analysis. Int. J. of Powder Met. and Powder Tech., Vol. 21, 2 (1985) 111-118.

68) Singh, P.N., Ramakrsihnan, P. and Beddow, J.K., Characterization of iron powders by Fourier analysis technique. In Proceedings of International Symposium on Recent Advances in Particulate Science and Technology, IIT Madras, 1982, pp 17-26.

69) Lew, G., Beddow, J.K. and Vetter, A.F., Morphological characterization of particle profiles derived from Walsh Coefficients. Powder Met. Int, 11 (1979) 165.

70) Meloy, T.P., Fast Fourier transforms applied to shape analysis of particle silhouettes to obtain morphological data. Powder Tech., 17 (1977) 27.

71) Okuda, Satoshi. and Takano, Hiroshi., Parameters for particle shape expression in Fast Fourier Transfer analysis. In Powder Metallurgy and Related High Temperature Materials. ed. P. Ramakrishnan, Oxford \& IBH Publishing Co., 1985, pp 47-68.

72) Beddow, J.K., Morphological analysis in particulate technology. Proceedings of International symposium on recent advances in Particulate Science and Technology, ed. Ramanujam, M. IIT, Madras 1982.

73) Kostelnik, M.C. and Beddow, J.K., as cited in Ref. 5. 
74) Kostelnik, M.C., Kludt. F.H. and Beddow, J.K., The initial stage of compaction of metal powders in a die. Int. J. Powder Met., 4 (1968) 19.

75) Riley, G.S. and Mann, G.R., Effects of particle shape on angles of repose and bulk densities of a granular solid. Mat. Res. Bull., 7 (1972) 163.
76) Paramanand. and Ramakrishnan, P.Effect of powder characteristics on compaction of parameters and ejection pressure of compacts. Powder Met., Vol. 27, 3 (1984) 163-168.

\section{Author's short biography}

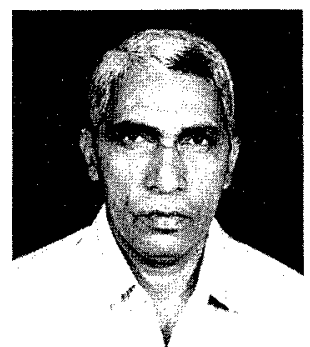

?

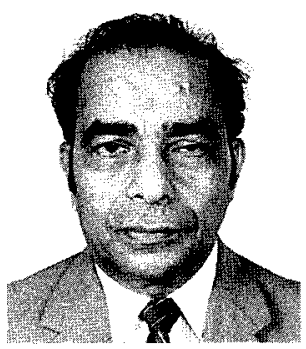

\section{Paramanand Singh}

Dr. Paramanand Singh graduated in Metallurgical Engineering in 1974, being awarded the gold medal for securing first rank. He received his M. Tech. in Nuclear Engineering and Technology from Indian Institute of Technology, Kanpur in 1976 and his $\mathrm{Ph}$. D. in 1984 from Indian Institute of Technology, Bombay. After completing his Ph. D. he conducted research and taught at IIT Bombay until 1985 when he was invited as a visiting scientist to join the group of Professor F. Thummler and Dr. S. Nazare at Kernforschungszentrum, Karlsruhe, F. R. G. researching in the area of high temperature intermetallic compounds for structural applicatons. He is with the Department of Metallurgical Engineering, IIT Madras since 1988 and is actively engaged in teaching, research and development, industrial consultancy and sponsored projects in the fields of powder metallurge, ceramics, composites and materials science in which he has published many papers in referred journals. He is on the editorial board of the Transactoin of Powder Metallurgy Association of India.

\section{P. Ramakrishnan}

Dr. P. Ramakrishnan, Professor Department of Metallurgical Eingineering and Materials Science, Chief Co-ordinator, Powder Metallurgy and High Temperature Material Centre, Indian Institute of Technology, Bombay is actively engaged in education, research and development, training and Industrial Consultancy in the field of Powder Metallurgy, Ceramic and Composite Materials. In these areas he has publihsed over 350 technical papers in reputed journals and edited 12 books. A recipient of Binani Gold Medal of Indian Insitute of Metals 1996, National Metallurgical Award of Government of India 1976, and the award of International Research Grant of National Science foundation U.S.A. on different occasions. He was the chairman of International Hausner award committee of the International fine particle society, U.S.A. in 1980, and the past president of PMAI, and Chairman of ASM International Chapter. He is a member of Technical Advisory Board, Editorial Advisory Board, Editorial Board and International Liaison Coommittee of many professional bodies, journals and International Conferences. 